\title{
Enantioselective Synthesis of Hydrobenzofuranones Using an Asymmetric Desymmetrizing Intramolecular Stetter Reaction of Cyclohexadienones
}

\author{
Qin Liu and Tomislav Rovis* \\ Department of Chemistry, Colorado State University \\ Fort Collins, Colorado 80523
}

\section{Supporting Information}

General Methods. All reactions were carried out under an atmosphere of argon in flame-dried glassware with magnetic stirring. Methanol was distilled from $\mathrm{CaH}_{2}$ prior to use. Methylene chloride was degassed with argon and passed through two column of neutral alumina. Toluene was degassed with argon and passed through one column of neutral alumina and one column of Q5 reactant. Column chromatography was performed on EM Science silica gel 60 (230-400 mesh). Thin layer chromatography was performed on EM Science $0.25 \mathrm{~mm}$ silica gel 60 -F plates. Visualization was accomplished with UV light, $\mathrm{KMnO}_{4}$, or aqueous ceric ammonium molybdate dips followed by heating.

KHMDS (0.5 M solution in toluene), Ethylene glycol (anhydrous, 99.8\%) and $\mathrm{PhI}(\mathrm{OAc})_{2}$ (iodobenzene diacetate, $98 \%$ ) was purchased from Aldrich Chemical Co. and used without purification.

${ }^{1} \mathrm{H}$ NMR was recorded at ambient temperature. Data are reported as follows: chemical shift in parts per million $(\delta, \mathrm{ppm})$ from deuterated chloroform $\left(\mathrm{CDCl}_{3}\right)$ or deuterated acetone (acetone-D6), multiplicity ( $\mathrm{s}=$ singlet, $\mathrm{d}=$ doublet, $\mathrm{t}=$ triplet, $\mathrm{q}=$ quartet, and $\mathrm{m}=$ multiplet), integration, and coupling constant $(\mathrm{Hz}) .{ }^{13} \mathrm{C} \mathrm{NMR}$ was recorded at ambient temperature. Chemical shifts are reported in ppm from $\left(\mathrm{CDCl}_{3}\right)$ taken as $77.0 \mathrm{ppm}$ or (acetone-D6) taken as 30.8 .

Full characterizations of 1, 2, 3, 4, 5, 6, 7, 8, 9, 11, 12, 13, 14, 15, 20, 27, 28, 29, 30, 31, $32,33,34,36,37,38,39,40,41,42$ have been reported in preliminary communication. ${ }^{[1]}$

Synthesis of tert-butyl 4-hydroxyphenethylcarbamate (starting material of substrate 10):
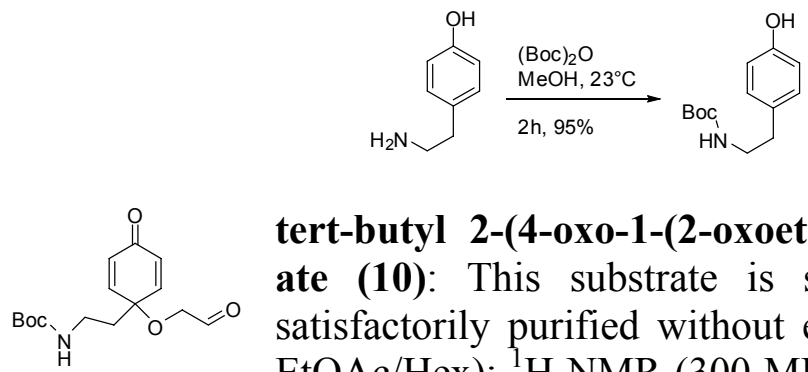

tert-butyl 2-(4-oxo-1-(2-oxoethoxy)cyclohexa-2,5-dienyl)ethylcarbam ate (10): This substrate is somewhat sensitive and could not be satisfactorily purified without extensive decomposition. $\mathrm{Rf}=0.23(2: 1$ EtOAc/Hex); ${ }^{1} \mathrm{H}$ NMR $\left(300 \mathrm{MHz}, \mathrm{CDCl}_{3}\right) \delta 9.64(\mathrm{~s}, 1 \mathrm{H}), 6.79(\mathrm{~d}, 1 \mathrm{H}, J$ $=10.2 \mathrm{~Hz}), 6.37(\mathrm{~d}, 1 \mathrm{H}, J=10.2 \mathrm{~Hz}), 4.84(\mathrm{~s}, 1 \mathrm{H}), 3.99(\mathrm{~s}, 2 \mathrm{H}), 3.26(\mathrm{dd}, 2 \mathrm{H}, J=6.6,6.5$

${ }^{[1]}$ Q. Liu, T. Rovis, Journal of the American Chemical Society 2006, 128, 2552. 
$\mathrm{Hz}), 2.05(\mathrm{t}, 2 \mathrm{H}, J=7.1 \mathrm{~Hz}), 1.44(\mathrm{~s}, 9 \mathrm{H}) ;{ }^{13} \mathrm{C} \mathrm{NMR}\left(100 \mathrm{MHz}, \mathrm{CDCl}_{3}\right) \delta 199.1,185.1$, 148.4, 132.1, 94.6, 71.0, 53.7, 39.8, 36.1, 28.6; IR (NaCl, neat) 3355, 2971, 2925, 1693, $1669,1167 \mathrm{~cm}^{-1}$; HRMS (EI+) calcd for $\mathrm{C}_{15} \mathrm{H}_{21} \mathrm{NO}_{5}, 295.1420$. Found 295.1419.

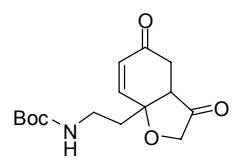

tert-butyl 2-(3,5-dioxo-2,3,3a,4,5,7a-hexahydrobenzofuran-7a-yl)ethyl carbamate (35): $\mathrm{Rf}=0.49(2: 1 \mathrm{EtOAc} / \mathrm{Hex}) ;[\alpha]_{\mathrm{D}}{ }^{21}=+39.6(\mathrm{c}=0.9$, $\mathrm{CHCl}_{3}$ ); HPLC analysis - Chiracel AD-H column 93:7 hexanes : isopropanol $1.0 \mathrm{~mL} / \mathrm{min}$. Major: 35.8 minutes, Minor: 29.8 minutes.; IR ( $\mathrm{NaCl}$, neat) 3360, 2971, 2925, 1766, 1788, $1168 \mathrm{~cm}^{-1}$; HRMS (FAB+) calcd for $\mathrm{C}_{15} \mathrm{H}_{21} \mathrm{O}_{5}, 295.1420$. Found 295.1419.

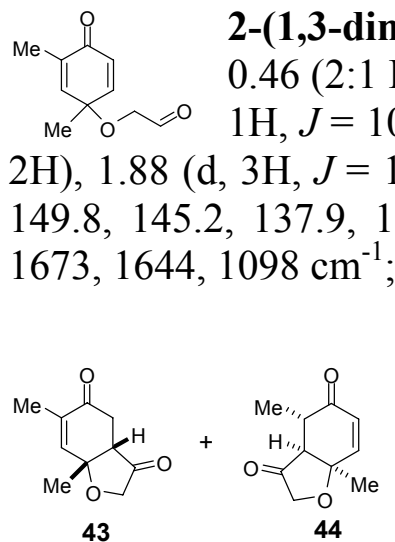

2-(1,3-dimethyl-4-oxocyclohexa-2,5-dienyloxy)acetaldehyde (16): $\mathrm{Rf}=$ 0.46 (2:1 EtOAc/Hex); ${ }^{1} \mathrm{H}$ NMR $\left(300 \mathrm{MHz}, \mathrm{CDCl}_{3}\right) \delta 9.61(\mathrm{~s}, 1 \mathrm{H}), 6.70(\mathrm{dd}$, $1 \mathrm{H}, J=10.0,3.0 \mathrm{~Hz}), 6.48-6.51(\mathrm{~m}, 1 \mathrm{H}), 6.27(\mathrm{~d}, 1 \mathrm{H}, J=10.0 \mathrm{~Hz}), 3.91(\mathrm{~s}$, 2H), $1.88(\mathrm{~d}, 3 \mathrm{H}, J=1.4 \mathrm{~Hz}), 1.49(\mathrm{~s}, 3 \mathrm{H}) ;{ }^{13} \mathrm{C} \mathrm{NMR}\left(75 \mathrm{MHz}, \mathrm{CDCl}_{3}\right) \delta 199.7,185.5$, 149.8, 145.2, 137.9, 130.8, 73.8, 71.3, 26.5, 16.1; IR (NaCl, neat) 2966, 2919, 1735, $1673,1644,1098 \mathrm{~cm}^{-1}$; HRMS (EI+) calcd for $\mathrm{C}_{10} \mathrm{H}_{12} \mathrm{O}_{3}, 180.0786$. Found 180.0786.

(3aS,7aR)-6,7a-dimethyl-3a,4-dihydrobenzofuran-3,5(2H, 7aH)-dione (43) and (3aS,4S,7aR)-4,7a-dimethyl-3a,4dihydrobenzofuran-3,5(2H,7aH)-dione (44): 43 and 44 cannot be separated. $\mathrm{Rf}=0.54(2: 1 \mathrm{EtOAc} / \mathrm{Hex}) ;[\alpha]_{\mathrm{D}}{ }^{21}=+61.2(\mathrm{c}=$ $0.9, \mathrm{CHCl}_{3}$, mixture of $\mathbf{4 3}$ and 44 with 2:1 ratio); Gas chromatography analysis - Chiraldex GTA column, gas flow $3 \mathrm{ml} / \mathrm{min}$ with constant 130 ${ }^{\circ} \mathrm{C}$ oven temperature. 43: Major: 9.6 min, Minor: 10.7 minutes; 44: Major: $10.3 \mathrm{~min}$, Minor: 13.4 minutes; IR (NaCl, neat) 2966, 2919, 2883, 1764, 1682, $1055 \mathrm{~cm}^{-1}$; HRMS (FAB+) calcd for $\mathrm{C}_{10} \mathrm{H}_{12} \mathrm{O}_{3}, 180.0786$. Found 180.0786 .

in 2-(1,2-dimethyl-4-oxocyclohexa-2,5-dienyloxy)acetaldehyde (17): $\mathrm{Rf}=$ 0.35 (2:1 EtOAc/Hex); ${ }^{1} \mathrm{H}$ NMR $\left(400 \mathrm{MHz}, \mathrm{CDCl}_{3}\right) \delta 9.63(\mathrm{~s}, 1 \mathrm{H}), 6.74(\mathrm{~d}$, $1 \mathrm{H}, J=10.0 \mathrm{~Hz}), 6.28(\mathrm{dd}, 1 \mathrm{H}, J=10.0,1.9 \mathrm{~Hz}), 6.19(\mathrm{~s}, 1 \mathrm{H}), 3.80(\mathrm{dd}, 2 \mathrm{H}$, $J=34.4,17.7 \mathrm{~Hz}), 1.98(\mathrm{~d}, 3 \mathrm{H}, J=1.0 \mathrm{~Hz}), 1.50(\mathrm{~s}, 3 \mathrm{H}) ;{ }^{13} \mathrm{C} \mathrm{NMR}\left(100 \mathrm{MHz}, \mathrm{CDCl}_{3}\right) \delta$ 198.9, 185.2, 158.9, 150.5, 130.7, 129.8, 75.2, 70.9, 25.3, 18.0; IR (NaCl, neat) 2981, $1735,1670,1635,1294,1093 \mathrm{~cm}^{-1}$; HRMS (FAB+) calcd for $\mathrm{C}_{10} \mathrm{H}_{12} \mathrm{O}_{3}, 180.0786$. Found 180.0786 .

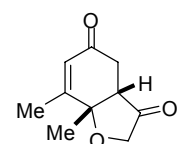

7,7a-dimethyl-3a,4-dihydrobenzofuran-3,5(2H,7aH)-dione (45): $\mathrm{Rf}=0.44$ $(2: 1 \mathrm{EtOAc} / \mathrm{Hex}) ;[\alpha]_{\mathrm{D}}{ }^{21}=+6.6\left(\mathrm{c}=1.5, \mathrm{CHCl}_{3}\right) ;$ Gas chromatography analysis - Chiraldex GTA column, gas flow $3 \mathrm{ml} / \mathrm{min}$ with constant $150{ }^{\circ} \mathrm{C}$ oven temperature. Major: $8.5 \mathrm{~min}$, Minor: 8.9 minutes; ${ }^{1} \mathrm{H}$ NMR $\left(300 \mathrm{MHz}, \mathrm{CDCl}_{3}\right) \delta$ $5.90(\mathrm{~s}, 1 \mathrm{H}), 4.17(\mathrm{dd}, 1 \mathrm{H}, J=17.2,1.1 \mathrm{~Hz}), 3.64(\mathrm{~d}, 1 \mathrm{H}, J=17.2 \mathrm{~Hz}), 3.01$ (ddd, $1 \mathrm{H}, J=$ $17.9,1.4,1.0 \mathrm{~Hz}), 2.69(\mathrm{~d}, 1 \mathrm{H}, J=7.1 \mathrm{~Hz}), 2.56(\mathrm{dd}, 1 \mathrm{H}, J=17.9,7.0 \mathrm{~Hz}), 1.98(\mathrm{~d}, 3 \mathrm{H}, J$ $=1.3 \mathrm{~Hz}), 1.68(\mathrm{~s}, 3 \mathrm{H}) ;{ }^{13} \mathrm{C} \mathrm{NMR}\left(75 \mathrm{MHz}, \mathrm{CDCl}_{3}\right) \delta 213.2,193.7,160.9,130.1,81.0$, 69.5, 52.2, 33.2, 23.8, 18.2; IR (NaCl, neat) 2981, 2914, 1760, 1683, 1270, $1052 \mathrm{~cm}^{-1}$; HRMS (FAB+) calcd for $\mathrm{C}_{10} \mathrm{H}_{12} \mathrm{O}_{3}, 180.0786$. Found 180.0786 . 




2-(7-oxo-1,2,3,4,4a,7-hexahydronaphthalen-4a-yloxy)acetaldehyde (18): $\mathrm{Rf}=0.38(2: 1 \mathrm{EtOAc} / \mathrm{Hex}) ;{ }^{1} \mathrm{H} \mathrm{NMR}\left(300 \mathrm{MHz}, \mathrm{CDCl}_{3}\right) \delta 9.65(\mathrm{~s}, 1 \mathrm{H}), 6.68$ $(\mathrm{d}, 1 \mathrm{H}, J=10.0 \mathrm{~Hz}), 6.31(\mathrm{dd}, 1 \mathrm{H}, J=10.0,1.9 \mathrm{~Hz}), 6.22(\mathrm{~s}, 1 \mathrm{H}), 3.79$ (dd, $2 \mathrm{H}, J=17.8,2.5 \mathrm{~Hz}), 2.23-2.44(\mathrm{~m}, 3 \mathrm{H}), 1.92-2.10(\mathrm{~m}, 2 \mathrm{H}), 1.60-1.72(\mathrm{~m}$, $1 \mathrm{H}), 1.28-1.48(\mathrm{~m}, 2 \mathrm{H}) ;{ }^{13} \mathrm{C} \mathrm{NMR}\left(75 \mathrm{MHz}, \mathrm{CDCl}_{3}\right) \delta 199.3,185.9,161.6,149.8,131.4$, 127.3, 74.4, 70.2, 39.2, 32.7, 28.1, 20.5; IR (NaCl, neat) 2939, 2855, 1734, 1664, 1092, $883 \mathrm{~cm}^{-1}$; HRMS (FAB+) calcd for $\mathrm{C}_{12} \mathrm{H}_{14} \mathrm{O}_{3}, 206.0943$. Found 206.0942.

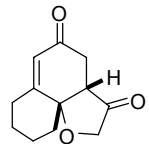

3a,4,7,8,9,10-hexahydro-2H-naphtho[1-b]furan-3,5-dione (46): $\mathrm{Rf}=0.56$ $(2: 1$ EtOAc/Hex $) ;[\alpha]_{\mathrm{D}}{ }^{21}=+2.3\left(\mathrm{c}=1.5, \mathrm{CHCl}_{3}\right) ;$ Gas chromatography analysis - Chiraldex B-DM column, gas flow $3 \mathrm{ml} / \mathrm{min}$ with constant $170{ }^{\circ} \mathrm{C}$ oven temperature. Major: $22.0 \mathrm{~min}$, Minor: 21.4 minutes; ${ }^{1} \mathrm{H} \mathrm{NMR}\left(300 \mathrm{MHz}, \mathrm{CDCl}_{3}\right) \delta$ $5.90(\mathrm{~s}, 1 \mathrm{H}), 4.15(\mathrm{dd}, 1 \mathrm{H}, J=17.2,0.7 \mathrm{~Hz}), 3.69(\mathrm{~d}, 1 \mathrm{H}, J=17.2 \mathrm{~Hz}), 2.97(\mathrm{~d}, 1 \mathrm{H}, J=$ $16.5 \mathrm{~Hz}), 2.62-2.75(\mathrm{~m}, 1 \mathrm{H}), 2.46-2.60(\mathrm{~m}, 2 \mathrm{H}), 1.90-2.26(\mathrm{~m}, 5 \mathrm{H}), 1.70-1.80(\mathrm{~m}, 1 \mathrm{H})$, $1.32-1.50(\mathrm{~m}, 1 \mathrm{H}) ;{ }^{13} \mathrm{C} \mathrm{NMR}\left(100 \mathrm{MHz}, \mathrm{CDCl}_{3}\right) \delta 214.3,194.6,163.7,126.6,80.9,68.9$, 50.6, 37.9, 34.2, 32.5, 28.1, 21.3; IR (NaCl, neat) 2940, 2858, 1762, 1675, 1245, 1050 $\mathrm{cm}^{-1}$; HRMS (FAB+) calcd for $\mathrm{C}_{12} \mathrm{H}_{14} \mathrm{O}_{3}, 206.0943$. Found 206.0942.

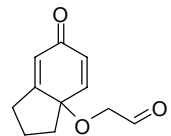

2-(6-oxo-2,3,3a,6-tetrahydro-1H-inden-3a-yloxy)acetaldehyde (19): $\mathrm{Rf}=$ $0.4(2: 1 \mathrm{EtOAc} / \mathrm{Hex}) ;{ }^{1} \mathrm{H}$ NMR $\left(300 \mathrm{MHz}, \mathrm{CDCl}_{3}\right) \delta 9.58(\mathrm{~s}, 1 \mathrm{H}), 6.80(\mathrm{~d}$, $1 \mathrm{H}, J=10.0 \mathrm{~Hz}), 6.28(\mathrm{dd}, 1 \mathrm{H}, J=9.9,1.7 \mathrm{~Hz}), 6.17-6.20(\mathrm{~m}, 1 \mathrm{H}), 3.81(\mathrm{dd}$, $2 \mathrm{H}, J=24.7,17.9 \mathrm{~Hz}), 2.61-2.78(\mathrm{~m}, 1 \mathrm{H}), 2.40-2.54(\mathrm{~m}, 1 \mathrm{H}), 2.18-2.34(\mathrm{~m}, 2 \mathrm{H}), 1.88-$ $2.22(\mathrm{~m}, 1 \mathrm{H}), 1.60-1.76(\mathrm{~m}, 1 \mathrm{H}) ;{ }^{13} \mathrm{C} \mathrm{NMR}\left(100 \mathrm{MHz}, \mathrm{CDCl}_{3}\right) \delta 199.4,185.9,165.5$, 144.0, 132.2, 125.9, 79.4, 69.9, 35.6, 28.8, 21.8; IR (NaCl, neat) 2955, 2838, 1733, 1668, $1645,1046 \mathrm{~cm}^{-1}$; HRMS (FAB+) calcd for $\mathrm{C}_{11} \mathrm{H}_{12} \mathrm{O}_{3}, 192.0786$. Found 192.0786.

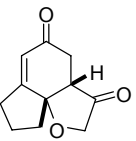

3a,4,8,9-tetrahydroindeno[4-b]furan-3,5(2H,7H)-dione (49): $\mathrm{Rf}=0.42(4: 1$

$\left.\mathrm{Et}_{2} \mathrm{O} / \mathrm{Hex}\right) ;[\alpha]_{\mathrm{D}}{ }^{21}=+13.2\left(\mathrm{c}=0.1, \mathrm{CHCl}_{3}\right) ;$ Gas chromatography analysis -

Chiraldex B-DM column, gas flow $3 \mathrm{ml} / \mathrm{min}$ with constant $150{ }^{\circ} \mathrm{C}$ oven temperature. Major: $29.7 \mathrm{~min}$, Minor: 27.0 minutes; ${ }^{1} \mathrm{H} \mathrm{NMR}\left(400 \mathrm{MHz}, \mathrm{CDCl}_{3}\right) \delta 6.02$ $(\mathrm{dd}, 1 \mathrm{H}, J=2.0,1.9 \mathrm{~Hz}), 4.20(\mathrm{dd}, 1 \mathrm{H}, J=17.5,0.8 \mathrm{~Hz}), 4.07$ (d, $1 \mathrm{H}, J=17.5), 2.76-$ $2.94(\mathrm{~m}, 2 \mathrm{H}), 2.46-2.64(\mathrm{~m}, 3 \mathrm{H}), 2.06-2.24(\mathrm{~m}, 2 \mathrm{H}), 1.84-1.96(\mathrm{~m}, 1 \mathrm{H}), 1.52-1.64(\mathrm{~m}$, $1 \mathrm{H}) ;{ }^{13} \mathrm{C}$ NMR $\left(75 \mathrm{MHz}, \mathrm{CDCl}_{3}\right) \delta 213.7,195.2,164.8,125.3,85.4,69.4,48.4,35.0$, 34.4, 29.6, 20.8; IR (NaCl, neat) 2955, 2843, 1757, 1675, 1035, $912 \mathrm{~cm}^{-1}$; HRMS (FAB+) calcd for $\mathrm{C}_{11} \mathrm{H}_{12} \mathrm{O}_{3}, 192.0786$. Found 192.0786.

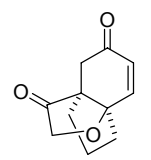

[4.3.3]oxo-propellenedione (50): $\mathrm{Rf}=0.48\left(4: 1 \mathrm{Et}_{2} \mathrm{O} / \mathrm{Hex}\right) ;[\alpha]_{\mathrm{D}}{ }^{21}=-112.7(\mathrm{c}$ $\left.=0.36, \mathrm{CHCl}_{3}\right)$; Gas chromatography analysis - Chiraldex B-DM column, gas flow $3 \mathrm{ml} / \mathrm{min}$ with constant $150{ }^{\circ} \mathrm{C}$ oven temperature. Major: $11.6 \mathrm{~min}$, Minor: 13.0 minutes; ${ }^{1} \mathrm{H}$ NMR $\left(400 \mathrm{MHz}, \mathrm{CDCl}_{3}\right) \delta 6.84(\mathrm{~d}, 1 \mathrm{H}, J=10.3 \mathrm{~Hz}), 6.02(\mathrm{~d}$, $1 \mathrm{H}, J=10.3 \mathrm{~Hz}), 4.30(\mathrm{~d}, 1 \mathrm{H}, J=17.3 \mathrm{~Hz}), 3.88(\mathrm{~d}, 1 \mathrm{H}, J=17.3 \mathrm{~Hz}), 3.06(\mathrm{~d}, 1 \mathrm{H}, J=$ $17.4 \mathrm{~Hz}), 2.36(\mathrm{~d}, 1 \mathrm{H}, J=17.4 \mathrm{~Hz}), 2.30-2.40(\mathrm{~m}, 1 \mathrm{H}), 1.94-2.18(\mathrm{~m}, 4 \mathrm{H}), 1.68-1.80$ (m, $1 \mathrm{H}) ;{ }^{13} \mathrm{C} \mathrm{NMR}\left(100 \mathrm{MHz}, \mathrm{CDCl}_{3}\right) \delta 215.6,195.4,147.4,130.3,90.7,69.9,57.9,41.2$, 37.8, 36.9, 24.4; IR (NaCl, neat) 2955, 2873, 1757, 1670, 1050, $917 \mathrm{~cm}^{-1}$; HRMS (FAB+) calcd for $\mathrm{C}_{11} \mathrm{H}_{12} \mathrm{O}_{3}, 192.0786$. Found 192.0786. 

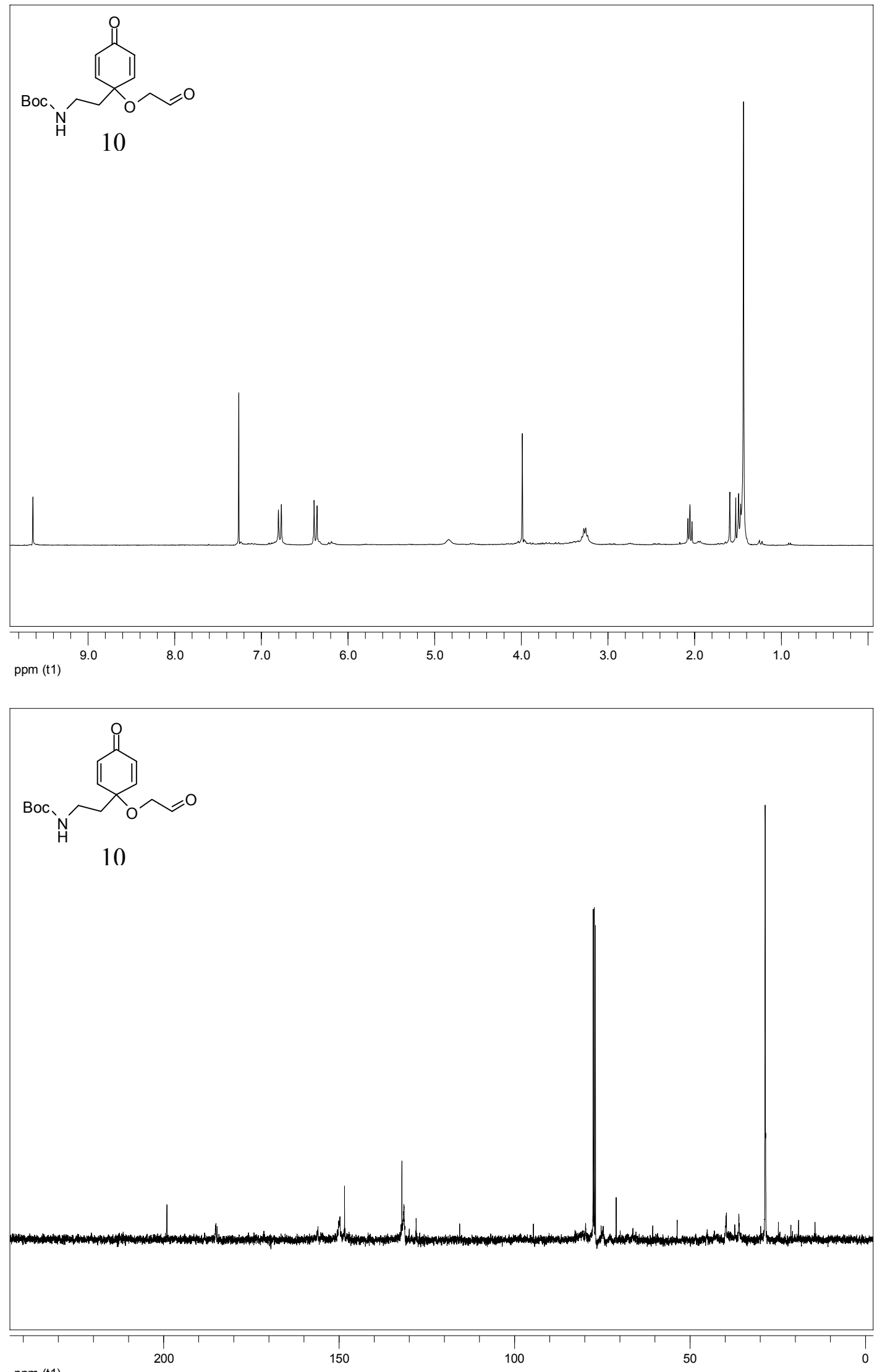

ppm (t1) 

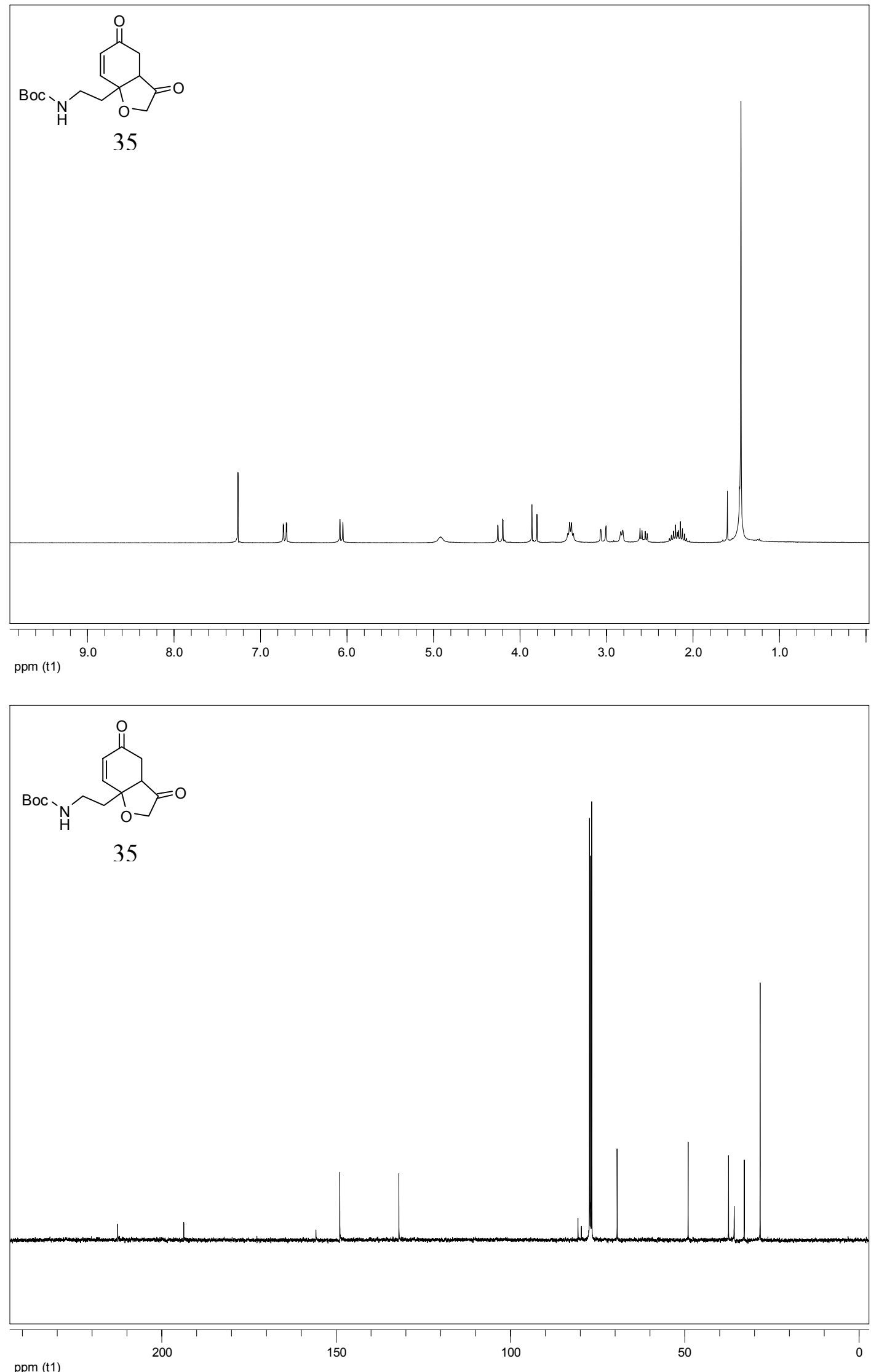

S-5 


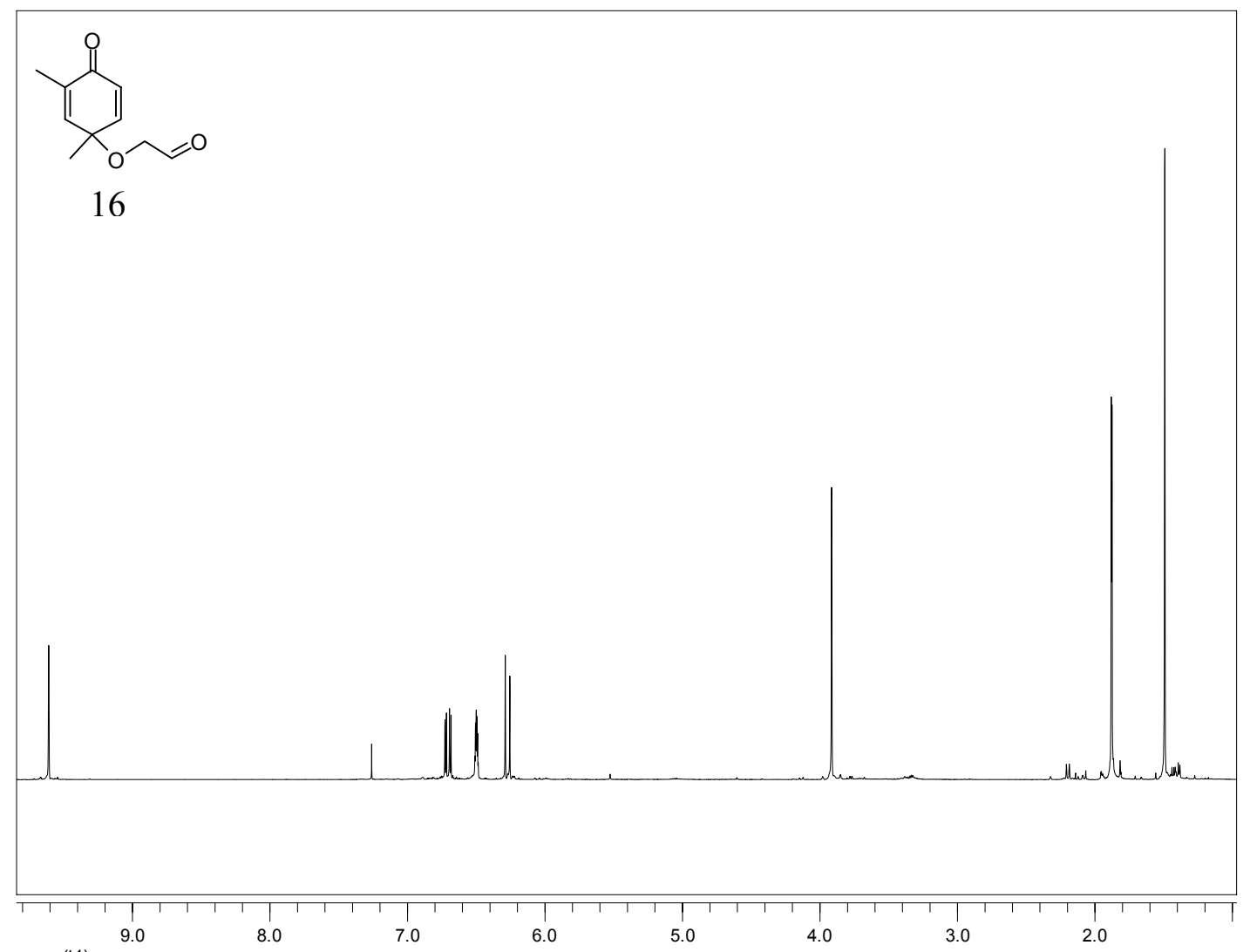

ppm (t1)

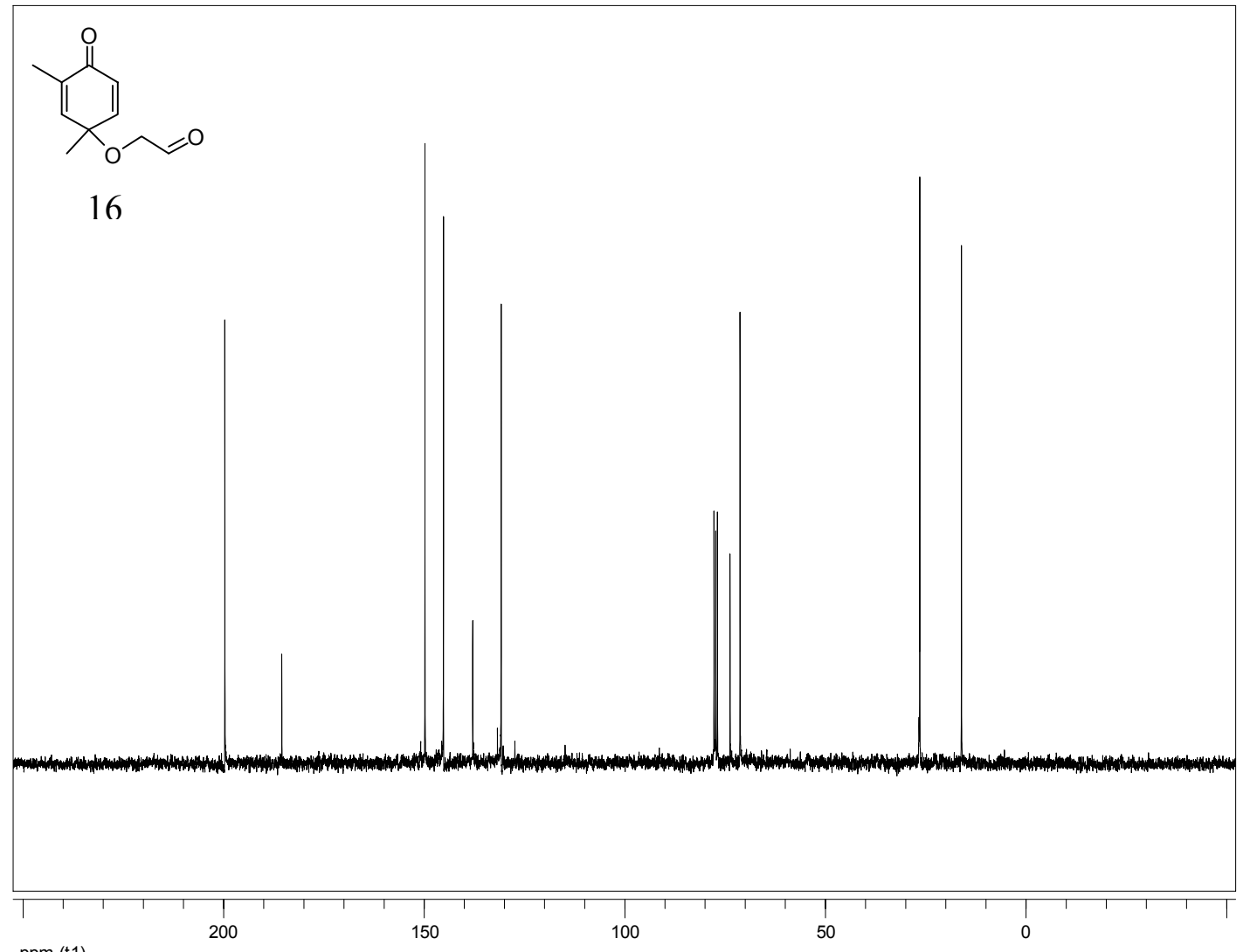

ppm (t1) 

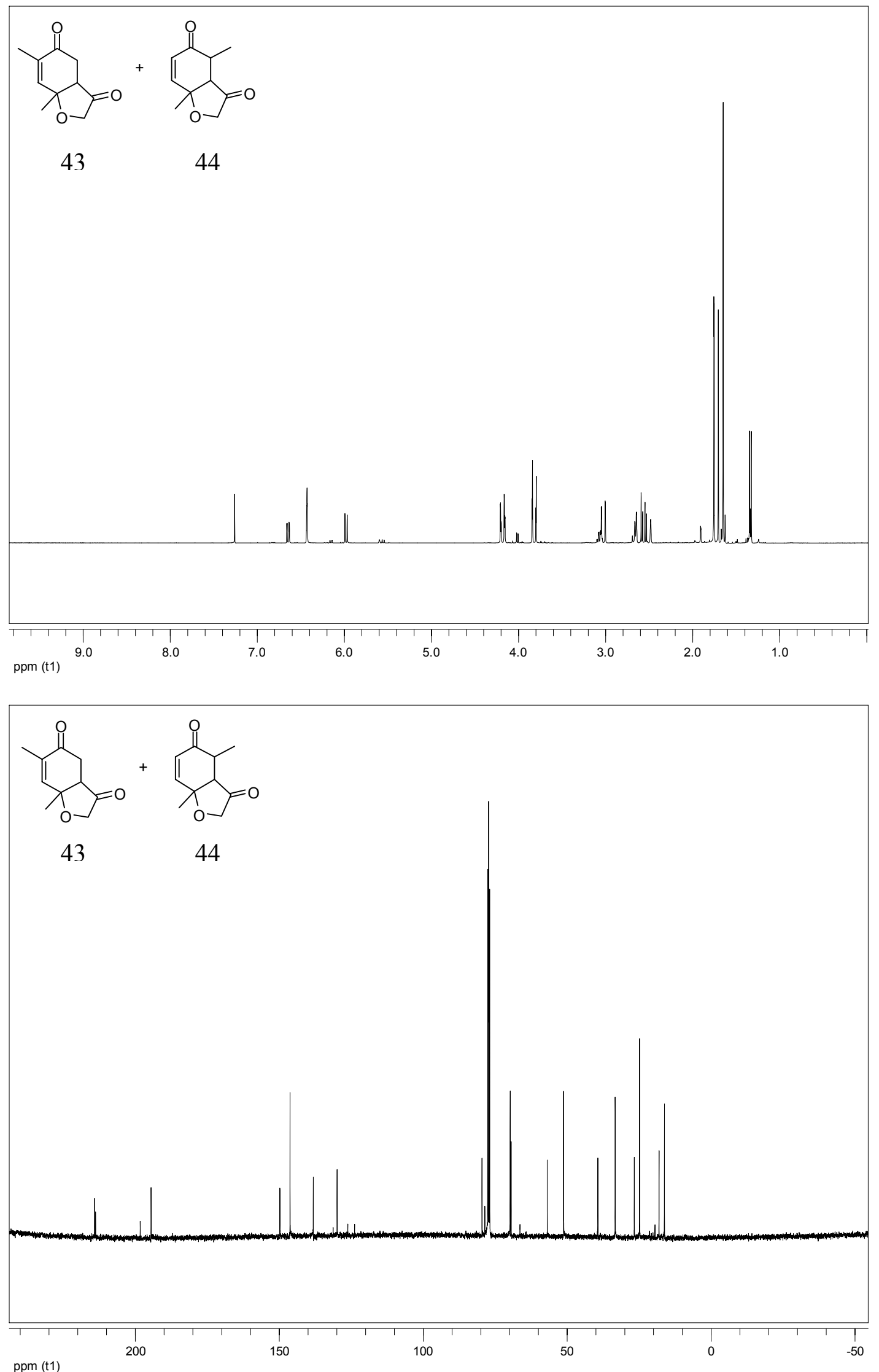

S-7 


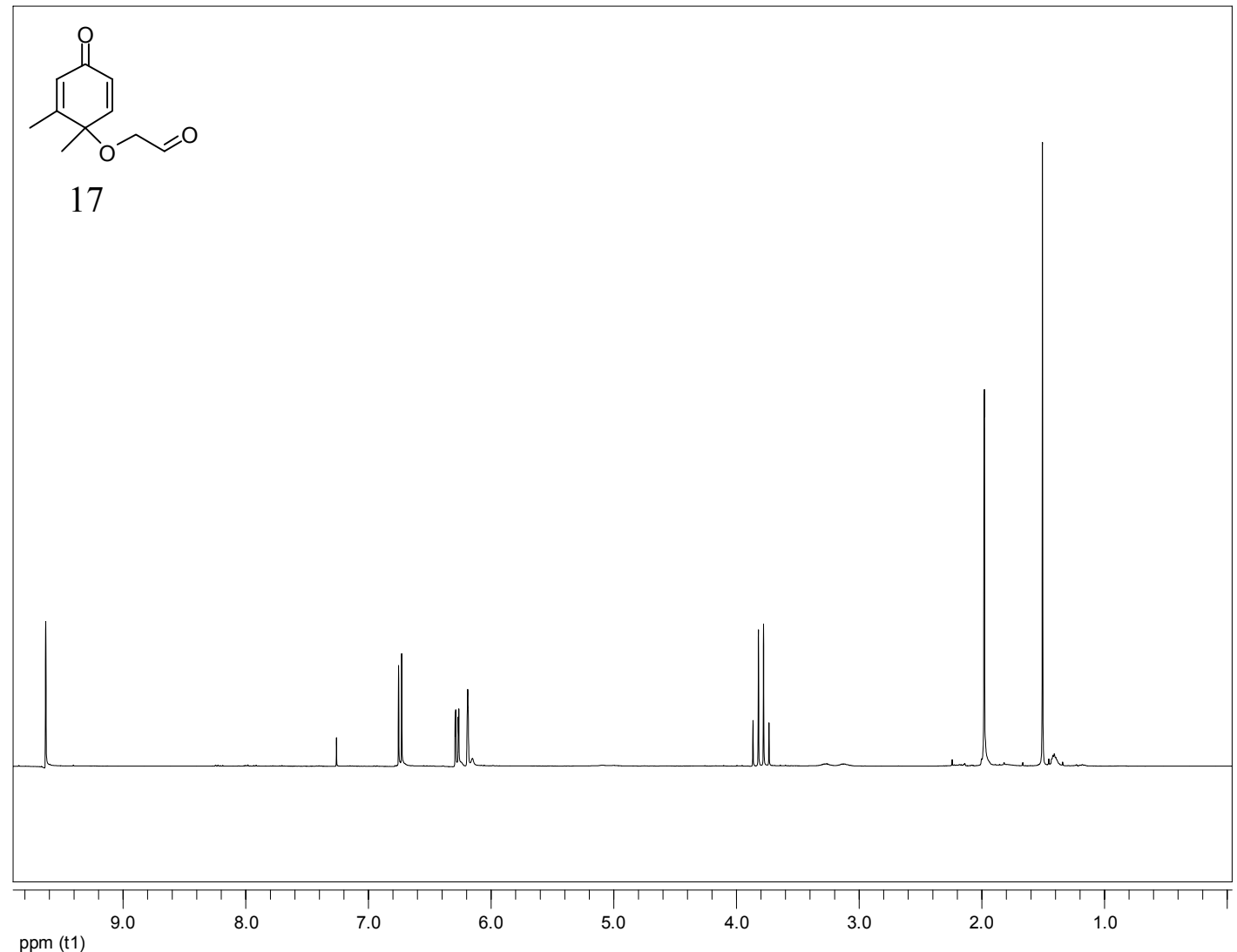
ppm (t1)

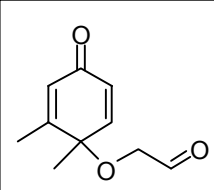

17
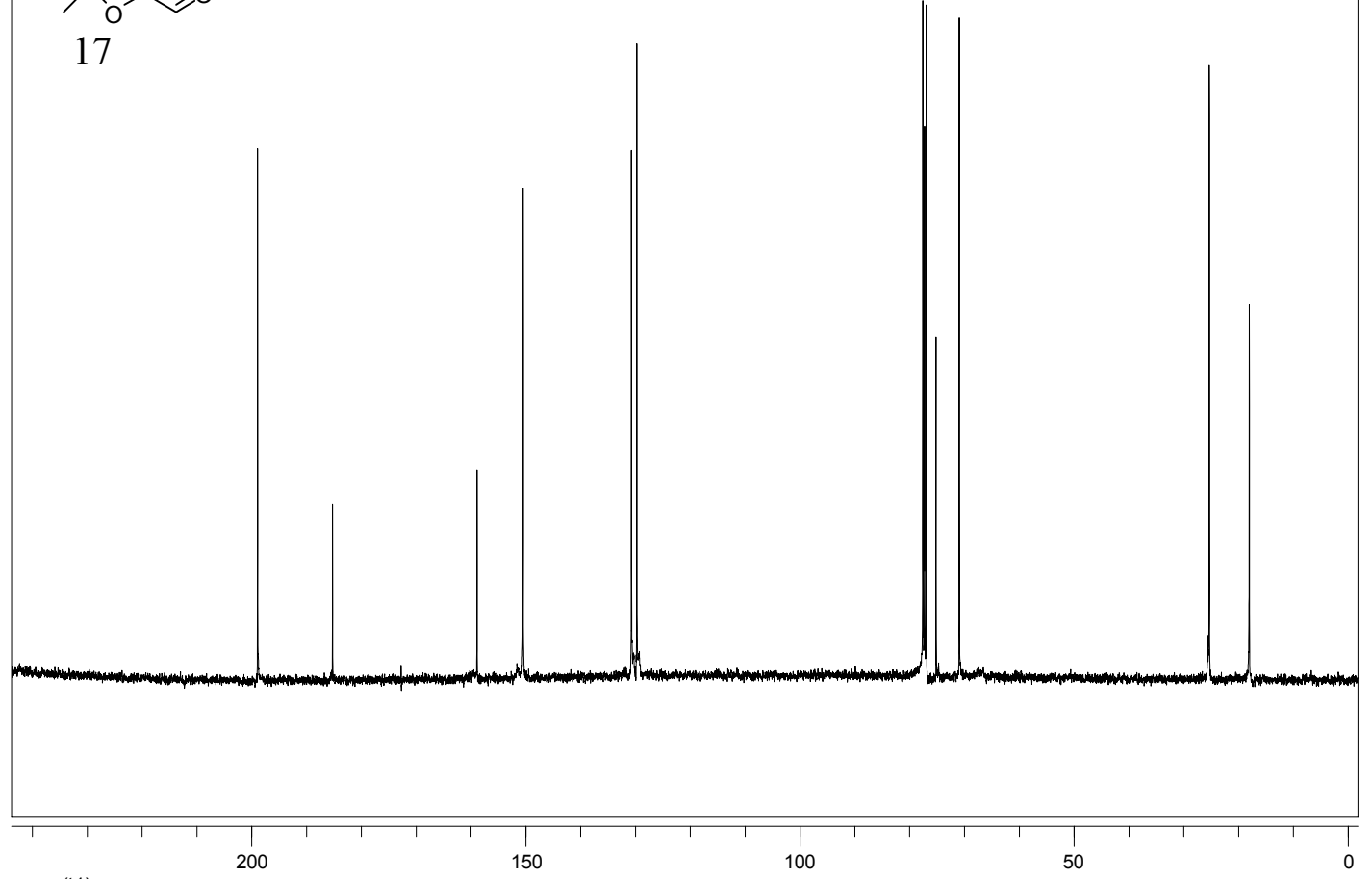

ppm (t1) 


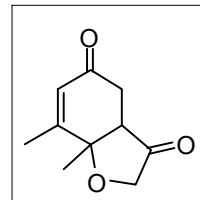

45

5
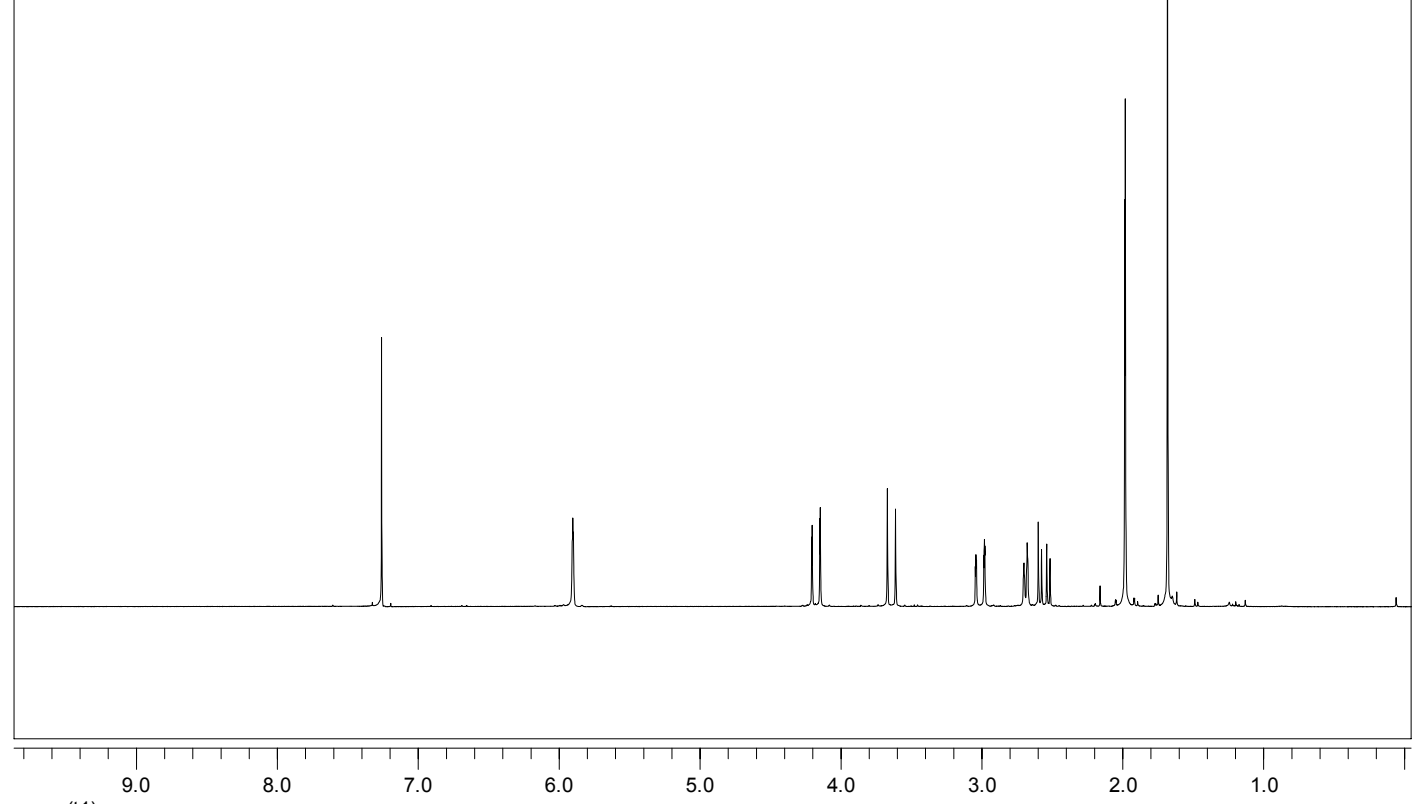

ppm (t1)

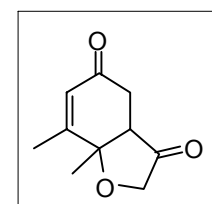

45
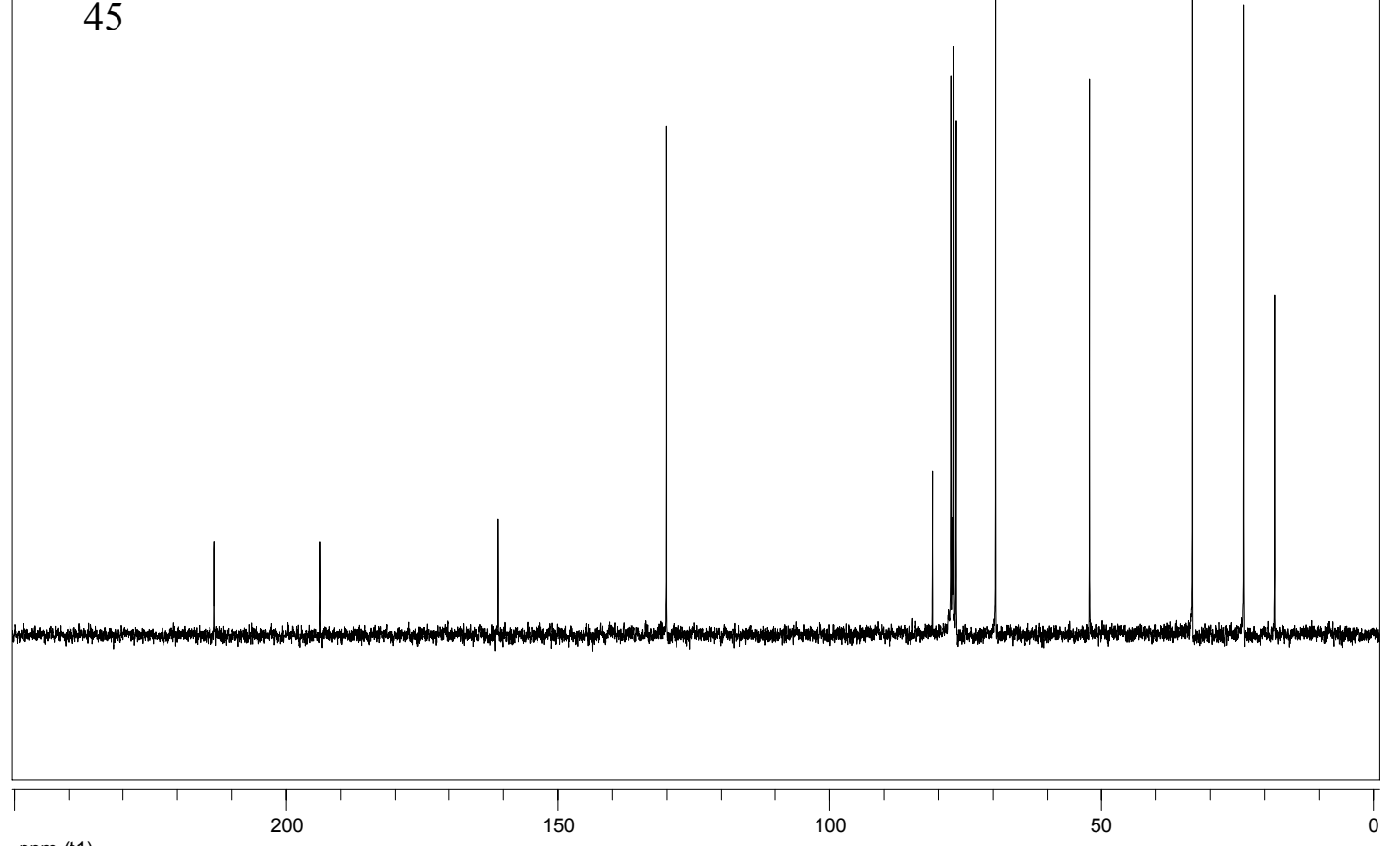

ppm (t1) 


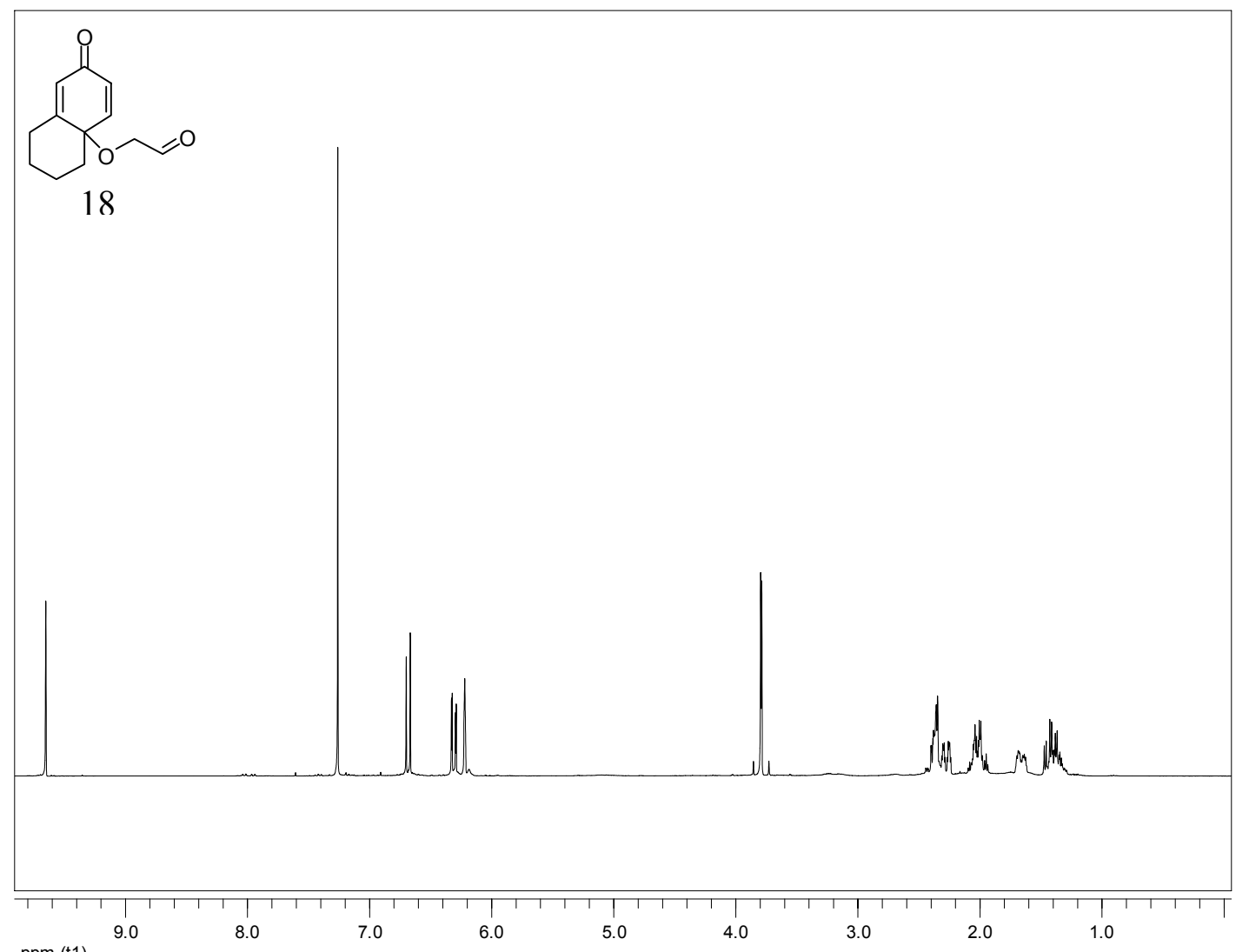

ppm (t1)

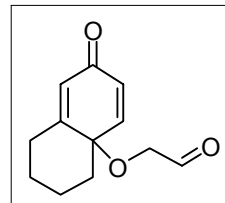

18


S-10 

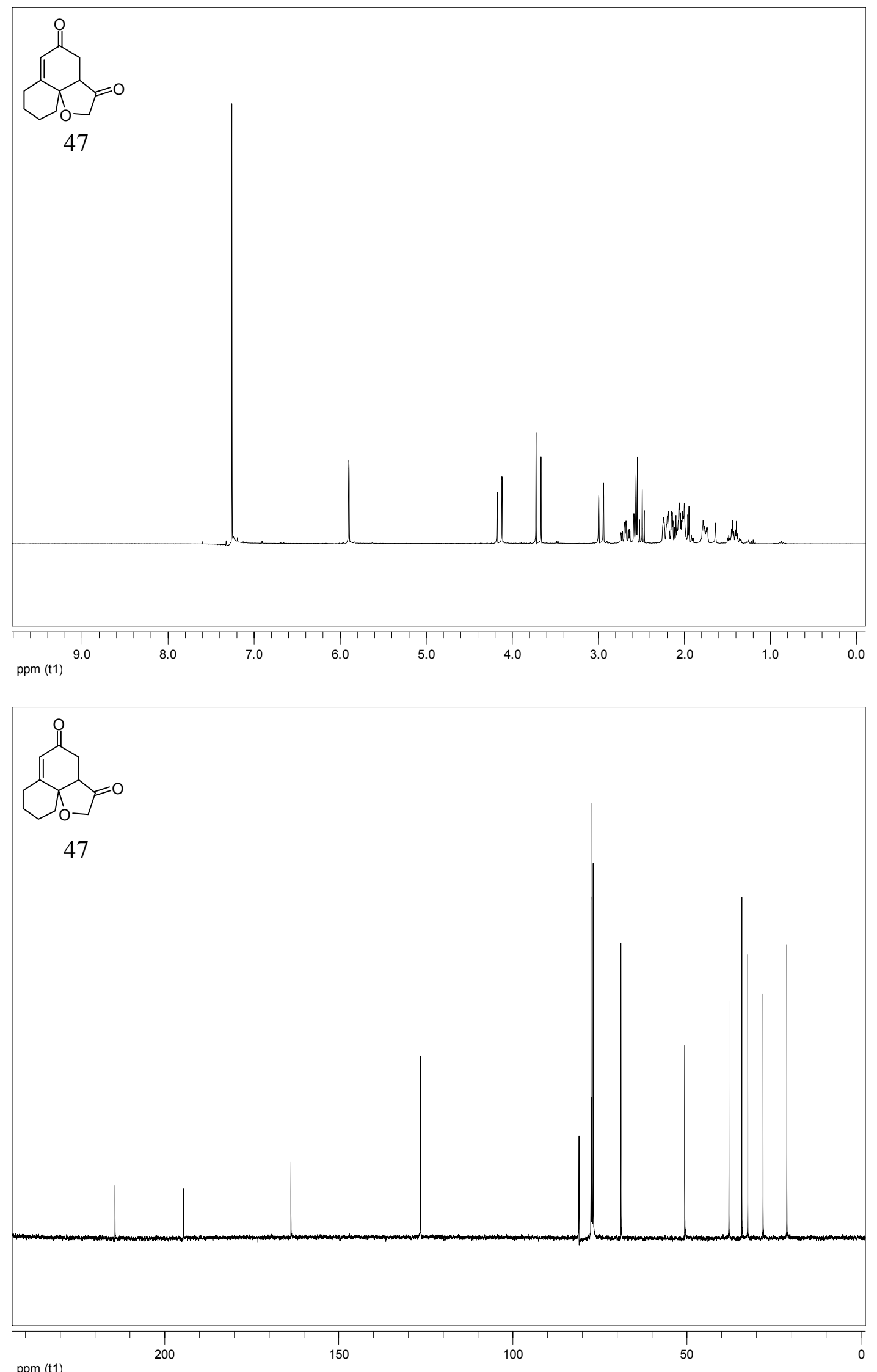

S-11 


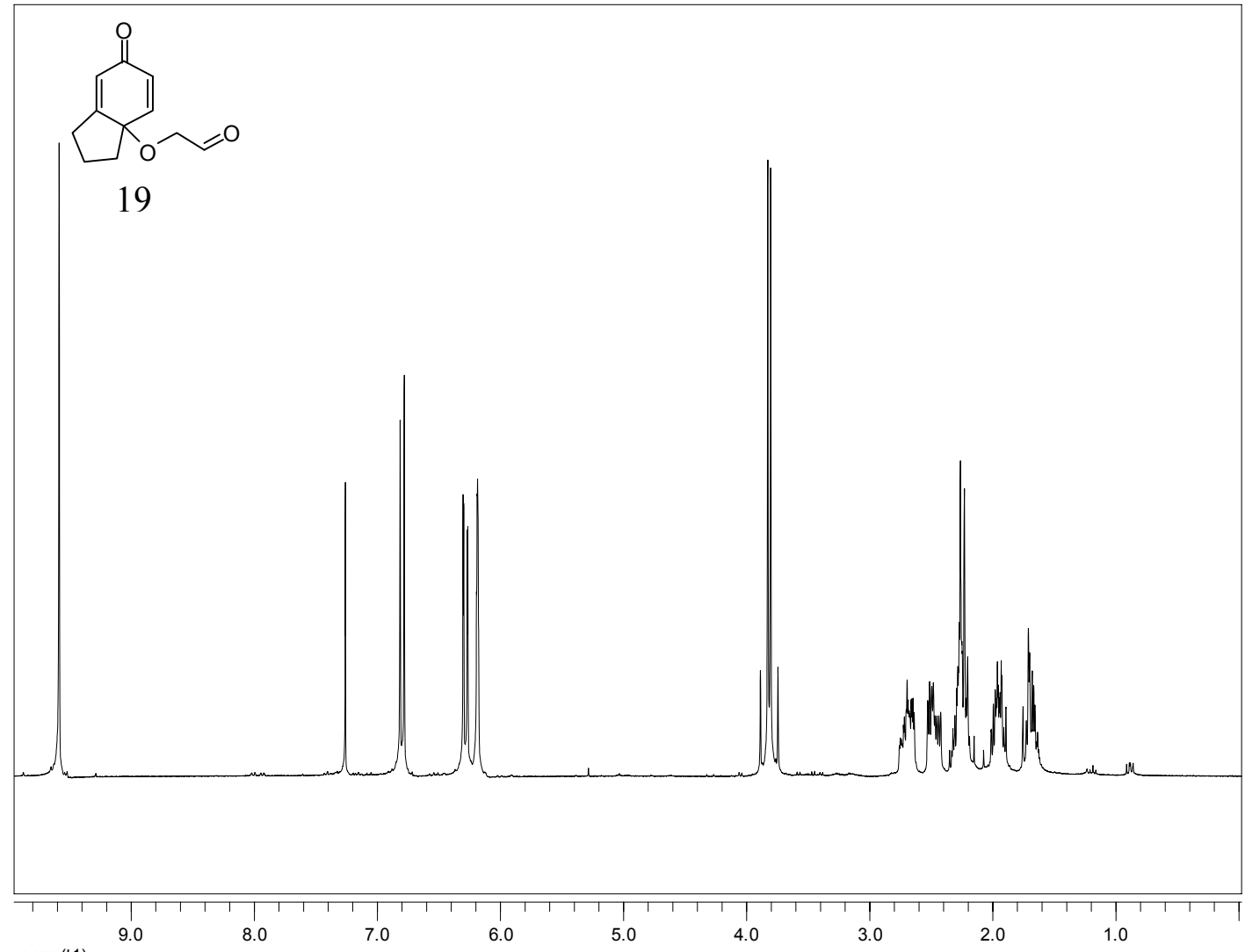

ppm (t1)

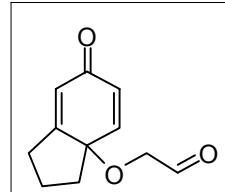

19


S-12 


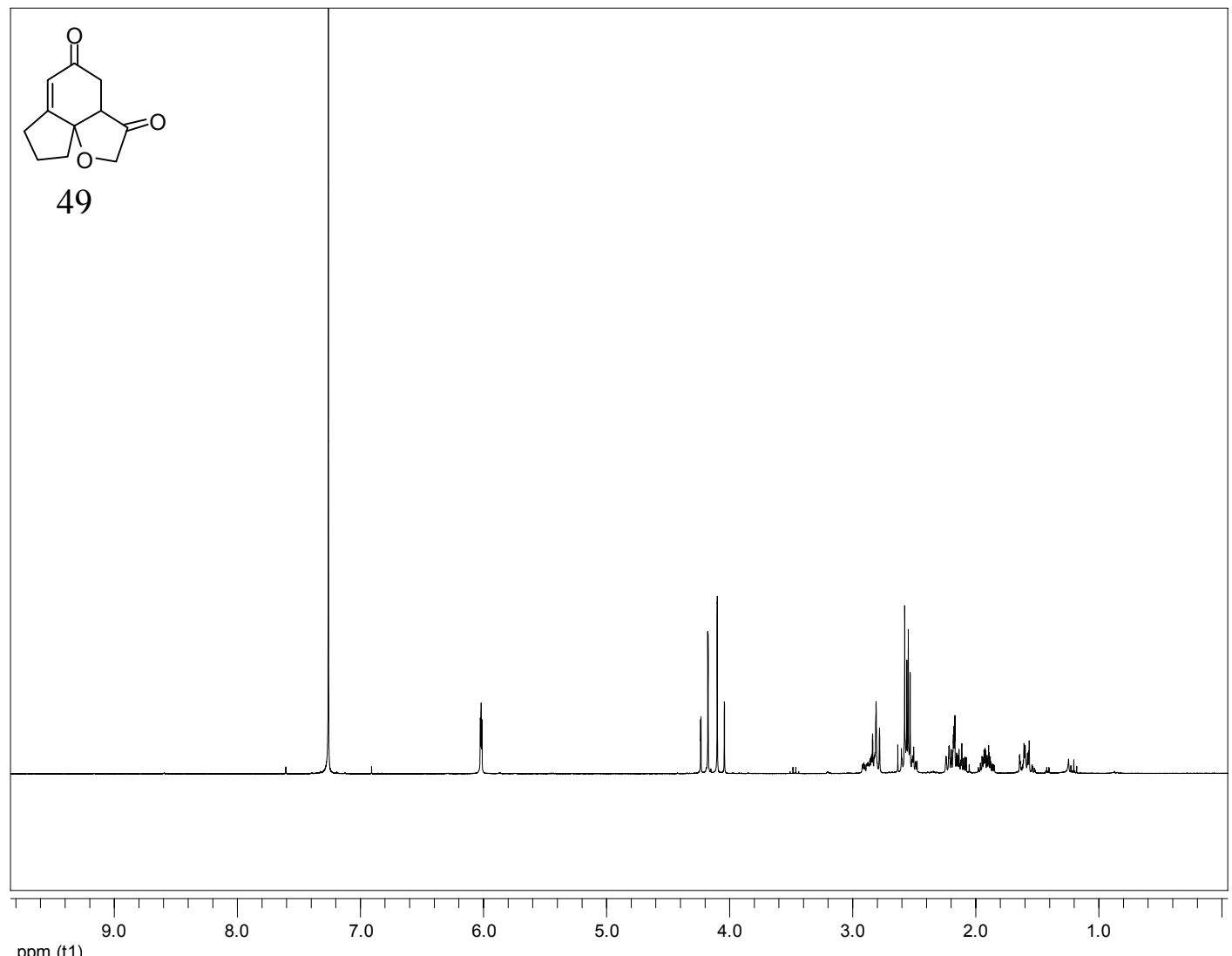

ppm (t1)

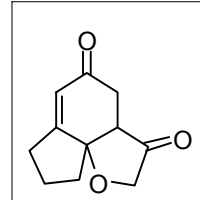

49

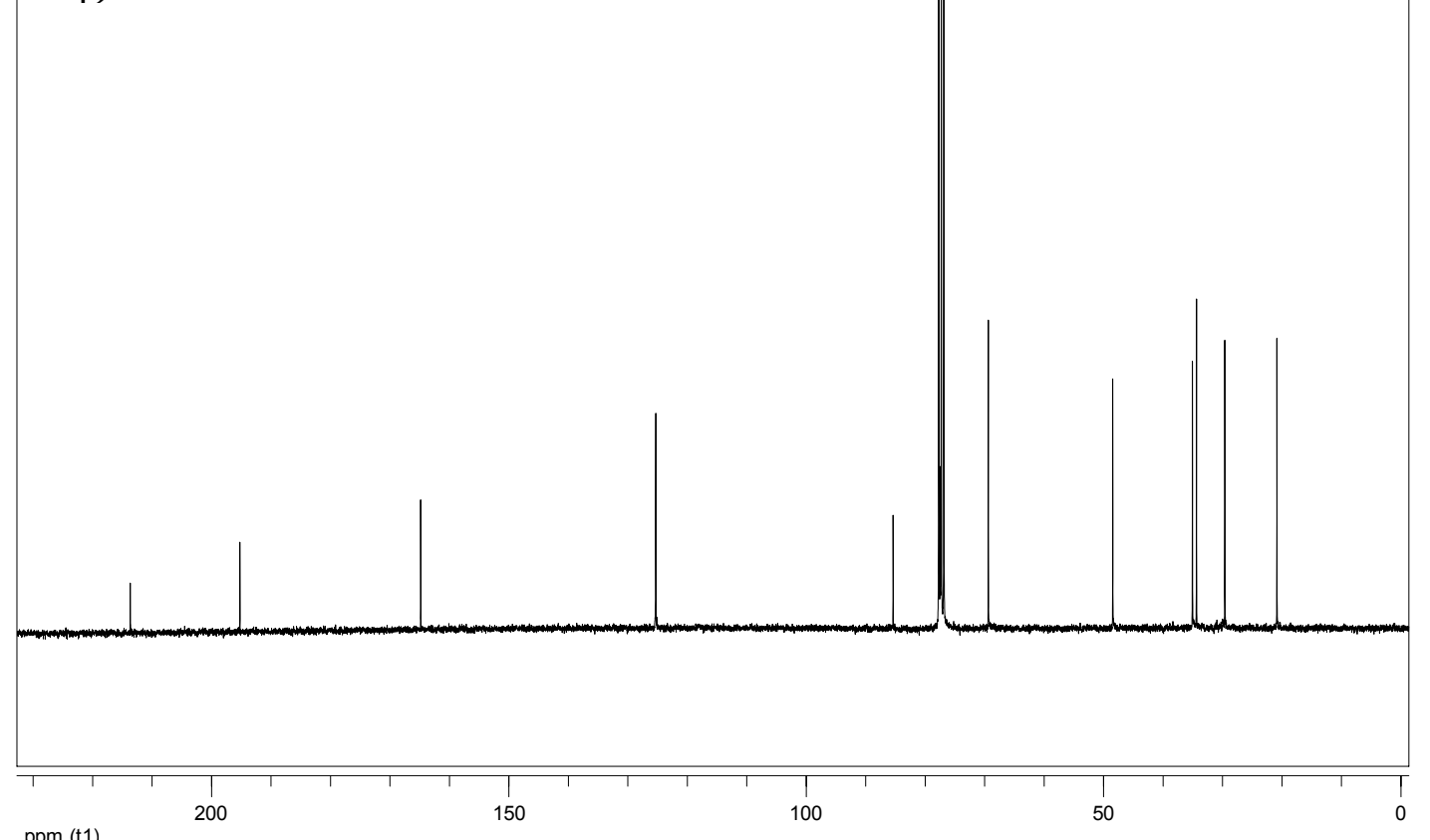

S-13 


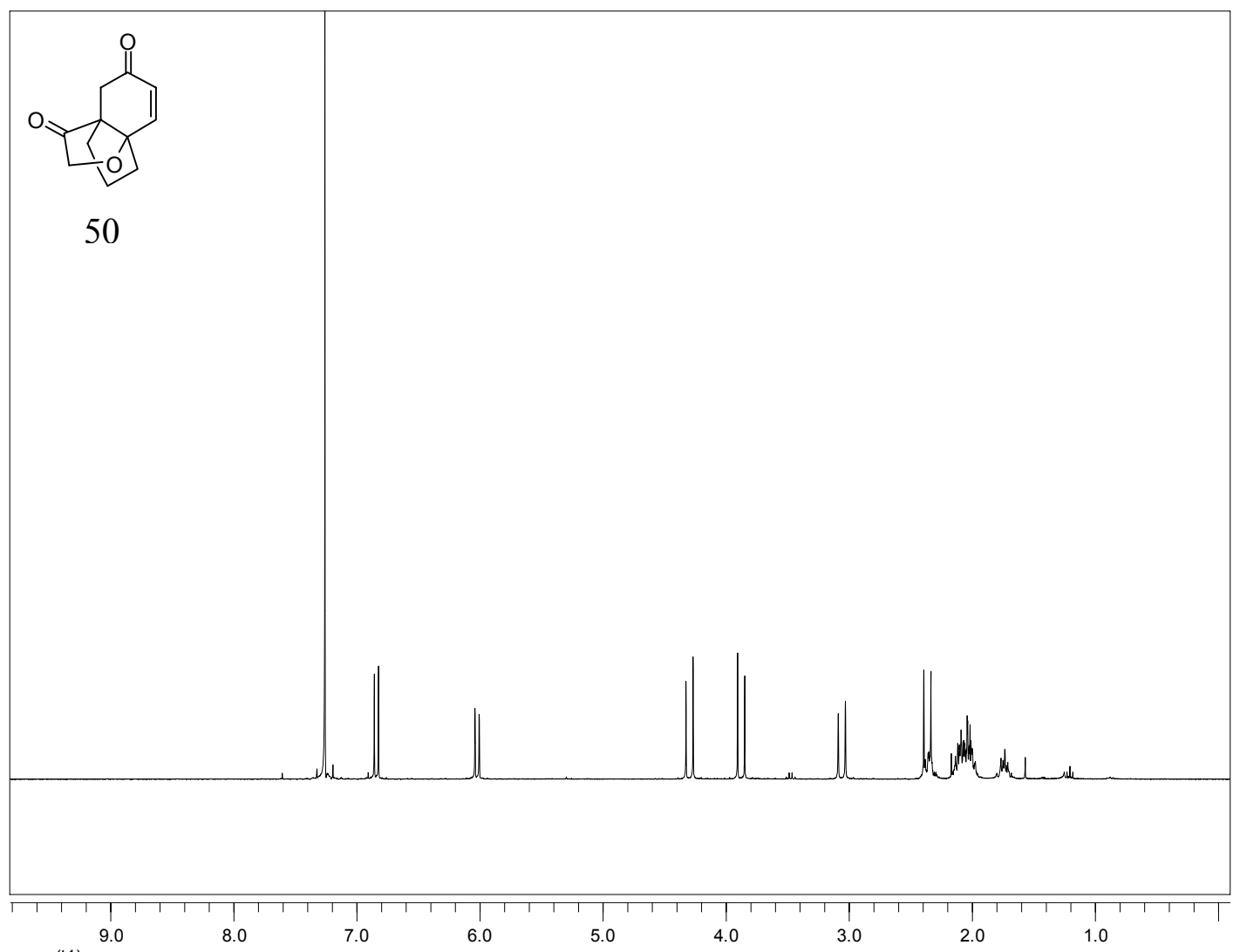

ppm (t1)



S-14 